\title{
Spectral Invariance of $*$-Representations of Twisted Convolution Algebras with Applications in Gabor Analysis
}

\author{
Are Austad ${ }^{1}$ \\ Received: 14 July 2020 / Revised: 26 January 2021 / Accepted: 6 April 2021 / \\ Published online: 4 June 2021 \\ (c) The Author(s) 2021
}

\begin{abstract}
We show spectral invariance for faithful $*$-representations for a class of twisted convolution algebras. More precisely, if $G$ is a locally compact group with a continuous 2-cocycle $c$ for which the corresponding Mackey group $G_{c}$ is $C^{*}$-unique and symmetric, then the twisted convolution algebra $L^{1}(G, c)$ is spectrally invariant in $\mathbb{B}(\mathcal{H})$ for any faithful $*$-representation of $L^{1}(G, c)$ as bounded operators on a Hilbert space $\mathcal{H}$. As an application of this result we give a proof of the statement that if $\Delta$ is a closed cocompact subgroup of the phase space of a locally compact abelian group $G^{\prime}$, and if $g$ is some function in the Feichtinger algebra $S_{0}\left(G^{\prime}\right)$ that generates a Gabor frame for $L^{2}\left(G^{\prime}\right)$ over $\Delta$, then both the canonical dual atom and the canonical tight atom associated to $g$ are also in $S_{0}\left(G^{\prime}\right)$. We do this without the use of periodization techniques from Gabor analysis.
\end{abstract}

Keywords Spectral invariance $\cdot$ Convolution algebras $\cdot C^{*}$-uniqueness $\cdot$ Gabor analysis

Mathematics Subject Classification $46 \mathrm{H} 15 \cdot 22 \mathrm{D} 15 \cdot 22 \mathrm{D} 20 \cdot 43 \mathrm{~A} 20 \cdot 43 \mathrm{~A} 70$

\section{Introduction}

The primary focus of this article is the concept of spectral invariance. In short, if $\mathcal{A}$ is a $*$-subalgebra of a Banach $*$-algebra $\mathcal{B}$, then $\mathcal{A}$ is said to be spectrally invariant in $\mathcal{B}$ if $\sigma_{\mathcal{A}}(a)=\sigma_{\mathcal{B}}(a)$ for all $a \in \mathcal{A}$, where $\sigma_{\mathcal{A}}(a)$ denotes the spectrum of the element $a$ in the algebra $\mathcal{A}$, and likewise for $\sigma_{\mathcal{B}}(a)$. In particular, if $\mathcal{A}$ and $\mathcal{B}$ are both unital

Communicated by Karlheinz Gröchenig.

$\triangle$ Are Austad

are.austad@ntnu.no

1 Department of Mathematical Sciences, Norwegian University of Science and Technology,

Trondheim, Norway 
with common unit, and if $a \in \mathcal{A}$ is invertible in $\mathcal{B}$, spectral invariance of $\mathcal{A}$ in $\mathcal{B}$ tells us that $a^{-1} \in \mathcal{A}$ as well. Spectral invariance of Banach $*$-algebras in $C^{*}$-algebras is a concept that has been extensively studied and is of importance in a number of different mathematical fields. Due to the seminal paper [22] the study of spectral invariance has been linked to Wiener's lemma, and variations of this result. As fields where spectral invariance is of importance we mention the theory of noncommutative tori $[9,30]$, Gabor analysis and window design in the theory of Gabor frames [30], convolution operators on locally compact groups $[4,19,20]$, infinite-dimensional matrices $[5,23$, $38,54]$, and the theory of pseudodifferential operators [26,27,31,54]. This list is by no means exhaustive. For an introduction to these variations on spectral invariance and Wiener's lemma we refer the reader to [28]. Moreover, we note that in recent years quite a bit of work has been done on spectral invariance of various algebras motivated by a plethora of different problems, see e.g. [6,29,45,46].

The main motivations for this article are the uses of spectral invariance in noncommutative geometry [10] and in Gabor analysis [30] as spectral invariance of twisted convolution algebras appear frequently in both. Indeed, Gabor analysis has in recent years been used as a source of examples for concepts in noncommutative geometry, see e.g. $[43,44]$. Our focus will not be on general $*$-subalgebras of Banach $*$-algebras. Instead we will limit ourselves to a subclass of all twisted convolution algebras of locally compact groups where the twist is implemented by a continuous 2-cocycle, see Definition 2.2. For such a locally compact group $G$ and a continuous 2-cocycle $c$, the resulting twisted convolution algebra will be denoted $L^{1}(G, c)$. Given a faithful $*$-representation $\pi: L^{1}(G, c) \rightarrow \mathbb{B}(\mathcal{H})$ for some Hilbert space $\mathcal{H}$, we wish to find conditions on $G$ and $\pi$ that guarantee that $\sigma_{L^{1}(G, c)}(f)=\sigma_{\mathbb{B}(\mathcal{H})}(\pi(f))$ for all $f \in L^{1}(G, c)$, i.e. that $L^{1}(G, c)$ is spectrally invariant in $\mathbb{B}(\mathcal{H})$. Key to our approach to this problem is the use of the Mackey group $G_{c}$ associated to the locally compact group $G$ and the continuous 2-cocycle $c$, and we define this group in Sect. 2.1. Note that in general $L^{1}(G, c)$ and $L^{1}\left(G_{c}\right)$ are not isomorphic as Banach $*$-algebras. It will be of importance to us that the convolution algebra $L^{1}\left(G_{c}\right)$ is symmetric, which in short means that the positive elements of the Banach $*$-algebra $L^{1}\left(G_{c}\right)$ have positive spectra, see Definition 2.6. We then apply Barnes' extension [4] of a result of Hulanicki [32], stated for the reader's convenience in Proposition 2.9, to prove the main result of the article.

Due to the use of the result of Hulanicki, the argument for spectral invariance will depend on a norm condition on self-adjoint elements. This norm condition may be difficult to check in practice, so we describe a class of groups for which the condition is automatically satisfied. This leads us to $C^{*}$-unique groups, introduced by Boidol [7]. In short, a locally compact group $G$ is $C^{*}$-unique if its convolution algebra $L^{1}(G)$ has a unique $C^{*}$-norm. A Banach $*$-algebra admitting a faithful $*$-representation is called $C^{*}$-unique if it has a unique $C^{*}$-completion. Examples of $C^{*}$-unique groups are semidirect products of abelian groups, connected metabelian groups, as well as groups where every compactly generated subgroup is of polynomial growth [7, p. 224]. We may now state the article's main theorem.

Theorem A (Theorem 3.1) Let $G$ be a locally compact group with a continuous 2cocycle $c$. 
(i) If $L^{1}\left(G_{c}\right)$ is $C^{*}$-unique, so is $L^{1}(G, c)$.

(ii) If $L^{1}\left(G_{c}\right)$ is symmetric and $C^{*}$-unique and $\pi: L^{1}(G, c) \rightarrow \mathbb{B}(\mathcal{H})$ is a faithful *-representation, then $f \mapsto\|\pi(f)\|_{\mathbb{B}(\mathcal{H})}, f \in L^{1}(G, c)$, is the full $C^{*}$-norm on $L^{1}(G, c)$, and $\sigma_{L^{1}(G, c)}(f)=\sigma_{\mathbb{B}(\mathcal{H})}(\pi(f))$ for all $f \in L^{1}(G, c)$.

Though there are some known examples of $C^{*}$-unique groups, there are very few statements in the literature concerning the $C^{*}$-uniqueness of twisted convolution algebras. This is why we go via the convolution algebra of the Mackey group $G_{c}$, and why statement $i$ ) is of independent interest. Note also that for all unital Banach $*$-algebras, being symmetric is equivalent to being spectrally invariant in the enveloping $C^{*}$-algebra, see for example [40, p. 340].

Important to our proof of the main theorem is the observation that convolution in $L^{1}\left(G_{c}\right)$ can be expressed in terms of convolution in the algebras $L^{1}\left(G, c^{n}\right), n \in \mathbb{Z}$, where $c^{n}$ is the 2-cocycle $c$ raised to the nth power, see Proposition 3.6. As an immediate consequence, $L^{1}\left(G_{c}\right)$ can be decomposed in terms of the subalgebras $L^{1}\left(G, c^{n}\right)$ as in Corollary 3.7, and this allows us to extend a faithful $*$-representation of $L^{1}(G, c)$ to a faithful $*$-representation of $L^{1}\left(G_{c}\right)$ in the proof of Theorem 3.1. This is the crucial step in the proof.

Using our main theorem we are able to give a short proof on a problem concerning regularity of canonical dual atoms and canonical tight atoms in Gabor analysis. We will do this by restating the problem in operator algebraic terms and then use Theorem 3.1. Exploring the interplay between Gabor analysis and operator algebras has gained much popularity in recent years $[2,3,14,35,37,43,44]$. The field of Gabor analysis has its origins in the seminal paper of Gabor [21], where he claimed that it is possible to obtain basis-like representations of functions in $L^{2}(\mathbb{R})$ in terms of the set $\left\{e^{2 \pi i l x} \phi(x-k)\right.$ : $k, l \in \mathbb{Z}$, where $\phi$ denotes a Gaussian. A central problem of the field is still to find basis-like expansions of functions in terms of time-frequency shifts of the form (4.1). Although most research in this field is done on one or several real variables, it is possible, due to the nature of time-frequency shifts, to study Gabor analysis on phase spaces of locally compact abelian groups [24]. Let $G$ be a locally compact abelian group. Then its phase space is the group $G \times \widehat{G}$, where $\widehat{G}$ is its Pontryagin dual. Let $\pi(z)$ be a time-frequency shift of the form (4.1) for some point $z=(x, \omega) \in G \times \widehat{G}$. Ignoring normalizations on the relevant Haar measures for the time being, one may then consider a closed, cocompact subgroup $\Delta \subseteq G \times \widehat{G}$ and a function $g \in L^{2}(G)$ and ask when a set $\mathcal{G}(g ; \Delta):=(\pi(z) g)_{z \in \Delta}$ is a frame for $L^{2}(G)$, i.e. when there exist constants $C, D>0$ for which

$$
C\|f\|_{2}^{2} \leq \int_{\Delta}|\langle f, \pi(z) g\rangle|^{2} \mathrm{~d} z \leq D\|f\|_{2}^{2}
$$

holds for all $f \in L^{2}(G)$, where $\mathrm{d} z$ is the chosen Haar measure on $\Delta$. The reason for assuming that $\Delta$ is cocompact will be explained in Remark 4.1. In time-frequency analysis it is often also of interest that the Gabor atom $g$ has good time-frequency decay. One way of expressing good time-frequency decay is to say that $g$ is in Feichtinger's algebra $S_{0}(G)$, see (4.5). 
Equivalent to $\mathcal{G}(g ; \Delta)$ being a Gabor frame for $L^{2}(G)$ is the invertibility of the frame operator $S: L^{2}(G) \rightarrow L^{2}(G)$ associated to $\mathcal{G}(g ; \Delta)$. The form of $S$ most suitable for our purposes is given in (4.6). Two functions of interest are then the canonical dual atom of $g$, which is $S^{-1} g$, and the canonical tight atom associated to $g$, which is $S^{-1 / 2} g$. They are of importance in Gabor analysis since they allow for perfect reconstuction formulas for all functions in $L^{2}(G)$ in terms of $g, S^{-1} g$, and $S^{-1 / 2} g$, as illustrated by (4.3) and (4.4). If $g \in S_{0}(G)$ generates a frame $\mathcal{G}(g ; \Delta)$ for $L^{2}(G)$, a natural question in Gabor analysis is then whether $S^{-1} g$ and $S^{-1 / 2} g$ are in $S_{0}(G)$ also. This leads us to our second main result.

Theorem B (Theorem 4.2) Let $\Delta \subseteq G \times \widehat{G}$ be a closed cocompact subgroup, and suppose $g \in S_{0}(G)$ is such that $\mathcal{G}(g ; \Delta)$ is a Gabor frame for $L^{2}(G)$. Then $S^{-1} g, S^{-1 / 2} g \in S_{0}(G)$ as well.

We note that the above result was proved in the case of separable lattices in $\mathbb{R}^{2 d}$, and claimed to hold more generally for lattices in phase spaces of locally compact abelian groups, in the celebrated paper [30]. Though it is somewhat technical to prove Theorem 3.1, our approach to Theorem 4.2 presented below makes it simple to prove the extension of the main result of [30] for general closed cocompact subgroups rather than just lattices. It may be possible to adapt the proof from [30] to this setting as well, but we offer a proof which makes no use of periodization techniques available in the setting of Gabor analysis.

As mentioned, to prove Theorem 4.2 we will restate the problem in operator algebraic language. For a Gabor frame $\mathcal{G}(g ; \Delta)$ with $g \in S_{0}(G)$, the frame operator $S$ can be rephrased in terms of a faithful (right) $*$-representation of the Banach $*$-algebra $\ell^{1}\left(\Delta^{\circ}, \bar{c}\right)$, where $\Delta^{\circ}$ is the adjoint lattice of $\Delta$ and $c$ is the Heisenberg 2-cocycle, see (4.2) and (4.7). As we explain in the proof of Theorem 4.2, any locally compact abelian group is $C^{*}$-unique and for any continuous 2-cocycle on it the associated Mackey group $G_{c}$ is also $C^{*}$-unique. In addition, $L^{1}\left(G_{C}\right)$ will in this case be symmetric. Hence we may apply Theorem 3.1 to obtain our second main result.

The strength in avoiding the periodization arguments of [30] and proving spectral invariance of a twisted $L^{1}$-algebra in terms of symmetry and $C^{*}$-uniqueness lies in the fact that the approach might be adaptable to other representations of groups where one has an analogous space to the Feichtinger algebra and an $L^{1}$-algebra acting on it, such as in the case of certain (projective) coorbit spaces $[8,15,16]$.

The article is organized as follows. Section 2 is dedicated to reminding the reader of some results on how we obtain twisted convolution algebras and $C^{*}$-algebras through projective unitary representations of locally compact groups, as well as some results on symmetric convolution algebras and $C^{*}$-unique groups. Our first main result is Theorem 3.1, and most of Sect. 3 is dedicated to the proof of this theorem, though some results are of independent interest. In Sect. 4 we rephrase a problem in Gabor analysis in terms of a faithful $*$-representation of a twisted convolution algebra, and apply Theorem 3.1 to obtain a simple proof of the main result of this section, Theorem 4.2. 


\section{Twisted Convolution Algebras}

\subsection{Projective Unitary Representations and Twisted Convolution Algebras}

We dedicate this section to explaining how we obtain twisted convolution algebras from projective unitary representations of locally compact groups.

Definition 2.1 Let $G$ be a locally compact group and let $\mathcal{U}(\mathcal{H})$ denote the group of unitary operators on the Hilbert space $\mathcal{H}$ equipped with the strong topology. A projective unitary representation of $G$ is a strongly continuous group homomorphism $\pi: G \rightarrow \mathcal{U}(\mathcal{H})$ satisfying

$$
\pi(e)=\operatorname{Id}_{\mathcal{H}}, \quad \pi\left(x_{1}\right) \pi\left(x_{2}\right)=c\left(x_{1}, x_{2}\right) \pi\left(x_{1} x_{2}\right)
$$

where $x_{1}, x_{2} \in G, e$ is the unit of $G$, and $c: G \times G \rightarrow \mathbb{T}$ is some continuous map.

The map $c: G \times G \rightarrow \mathbb{T}$ associated to the projective group representation $\pi: G \rightarrow$ $\mathcal{U}(\mathcal{H})$ in Definition 2.1 has some important properties. Using associativity of $\pi$ we realize that

$$
c\left(x_{1}, x_{2}\right) c\left(x_{1} x_{2}, x_{3}\right)=c\left(x_{1}, x_{2} x_{3}\right) c\left(x_{2}, x_{3}\right), \quad x_{1}, x_{2}, x_{3} \in G .
$$

Moreover, $\pi(e)=\operatorname{Id}_{\mathcal{H}}$ forces

$$
c(x, e)=c(e, x)=1, \quad x \in G .
$$

Definition 2.2 Let $G$ be a locally compact group. A continuous map $c: G \times G \rightarrow \mathbb{T}$ satisfying (2.1) and (2.2) is called a continuous 2-cocycle for $G$.

Continuous 2-cocycles are part of a cohomology theory for groups, though this is not something we will have much need for in the sequel. The following result lists some elementary results for 2-cocycles of groups.

Lemma 2.3 For a continuous 2-cocycle c for a locally compact group $G$ we have

(i) For any $n \in \mathbb{Z}$, the map $c^{n}: G \times G \rightarrow \mathbb{T}$ given by

$$
c^{n}\left(x_{1}, x_{2}\right)=\left(c\left(x_{1}, x_{2}\right)\right)^{n}, \quad x_{1}, x_{2} \in G,
$$

is also a continuous 2-cocycle.

(ii) For all $x \in G$ we have

$$
c\left(x, x^{-1}\right)=c\left(x^{-1}, x\right) .
$$

(iii) For all $x, y \in G$ we have

$$
c\left(y, y^{-1}\right) \overline{c\left(y^{-1}, x\right)}=c\left(y, y^{-1} x\right)
$$


Proof Statement i) is obvious. Statement ii) follows by setting $x_{1}=x_{3}=x$ and $x_{2}=x^{-1}$ in (2.1) and then using (2.2). For iii) we may equivalently show that

$$
c\left(y, y^{-1}\right) c\left(y y^{-1}, x\right)=c\left(y, y^{-1} x\right) c\left(y^{-1}, x\right)
$$

since $c\left(y y^{-1}, x\right)=1$. Setting $x_{1}=y, x_{2}=y^{-1}$ and $x_{3}=x$ in (2.1) and then using (2.2) we obtain the result.

Given a locally compact group $G$ and a continuous 2-cocycle $c$ for $G$, there is always a distinguished $c$-projective unitary representation of $G$, namely the $c$-twisted left regular representation. It is the map $L^{c}: G \rightarrow \mathcal{U}\left(L^{2}(G)\right)$ given by

$$
L_{y}^{c} f(x)=c\left(y, y^{-1} x\right) f\left(y^{-1} x\right), \quad x, y \in G, f \in L^{2}(G) .
$$

If $c=1$ we drop the $c$ from the notation and just write $L_{y}$ for $y \in G$.

Given a locally compact group $G$ and a continuous 2-cocycle $c$, we can construct an associated group $G_{c}$ known as the Mackey group. It has appeared in the literature numerous times before. As a topological space, $G_{c}$ is just $G \times \mathbb{T}$ with the product topology. The binary operation is given by

$$
\left(x_{1}, \tau_{1}\right)\left(x_{2}, \tau_{2}\right)=\left(x_{1} x_{2}, \tau_{1} \tau_{2} \overline{c\left(x_{1}, x_{2}\right)}\right) .
$$

The identity is given by $(e, 1)$, and the inverse of an element $(x, \tau) \in G_{c}$ is given by $(x, \tau)^{-1}=\left(x^{-1}, \bar{\tau} c\left(x^{-1}, x\right)\right) . G_{c}$ is a locally compact group, and its left Haar measure is the product measure. Hence its modular function may be identified with the modular function of $G$. We normalize the measure of $\mathbb{T}$ to 1 .

The usefulness of the Mackey group for us is in the fact that $c$-projective unitary representations of $G$ induce unitary representations of $G_{c}$. Explicitly, given a $c$-projective unitary representation of $G$, say $\pi: G \rightarrow \mathcal{U}(\mathcal{H})$ for some Hilbert space $\mathcal{H}$, we obtain a unitary representation $\pi_{c}: G_{c} \rightarrow \mathcal{U}(\mathcal{H})$ by setting

$$
\pi_{c}(x, \tau)=\bar{\tau} \pi(x)
$$

for $(x, \tau) \in G_{c}$.

We proceed to introduce twisted convolution algebras of these groups and show how we may complete them to $C^{*}$-algebras. For a locally compact group $G$ with modular function $m$, we consider the space of measurable and integrable functions $L^{1}(G)$. For a continuous 2-cocycle $c$ for $G$ we define $c$-twisted convolution on $L^{1}(G)$ by

$$
f_{1} \natural_{c} f_{2}(x)=\int_{G} f_{1}(y) f_{2}\left(y^{-1} x\right) c\left(y, y^{-1} x\right) \mathrm{d} y,
$$

for $f_{1}, f_{2} \in L^{1}(G)$, where $\mathrm{d} y$ is the Haar measure on $G$. Should $f_{2} \in L^{p}(G)$ and $p \in[1, \infty]$ we will use the same notation. We also define the $c$-twisted involution

$$
f^{* c}(x)=m\left(x^{-1}\right) \overline{c\left(x, x^{-1}\right) f\left(x^{-1}\right)}
$$


for $f \in L^{1}(G)$. We denote the resulting $*$-algebra by $L^{1}(G, c)$. It becomes a Banach *-algebra when equipped with the usual $L^{1}$-norm.

Any $c$-projective unitary representation $\pi: G \rightarrow \mathcal{U}(\mathcal{H})$ now induces a nondegenerate $*$-representation $\pi: L^{1}(G, c) \rightarrow \mathbb{B}(\mathcal{H})$ by setting

$$
\pi(f) \eta=\int_{G} f(x) \pi(x) \eta \mathrm{d} x, \quad f \in L^{1}(G, c), \eta \in \mathcal{H},
$$

where we interpret the integral weakly in $\mathcal{H}$. Note that $\|\pi(f)\| \leq\|f\|_{L^{1}(G)}$. If the integrated representation $\pi$ is faithful this gives us a way of completing $L^{1}(G, c)$ to a $C^{*}$-algebra, namely for any $f \in L^{1}(G)$ we set $\|f\|:=\|\pi(f)\|_{\mathbb{B}(\mathcal{H}) \text {. The integrated }}$ representation of the $c$-twisted left regular representation will be denoted by $f \mapsto L_{f}^{c}$. The following result, which will be important for us in the proof of Theorem 3.1, is a special case of [39, Satz 6].

Proposition 2.4 Let $G$ be an amenable locally compact group with a continuous 2cocycle c. Then $f \mapsto\left\|L_{f}^{c}\right\|_{\mathbb{B}\left(L^{2}(G)\right)}$ is the maximal $C^{*}$-norm on $L^{1}(G, c)$.

Instead of twisting the convolution algebra of the locally compact group $G$ by a continuous 2-cocycle $c$, we could first "twist" the group $G$ by $c$ to obtain the associated Mackey group $G_{c}$, and then consider the associated convolution algebra $L^{1}\left(G_{c}\right)$ with usual (untwisted) convolution and involution. We will have much use for this in the sequel. Any $c$-projective unitary representation of $G$ induces a unitary representation $\pi_{c}$ of $G_{c}$ by (2.5), which in turn induces a nondegenerate $*$-representation $\pi_{c}$ of $L^{1}\left(G_{c}\right)$. Note however that $\pi_{c}$ is in general not a faithful $*$-representation of $L^{1}\left(G_{c}\right)$ even if $\pi_{c}$ is a faithful unitary representation of $G_{c}$. Indeed, let $f \in L^{1}(G) \backslash\{0\}$ and define $F \in L^{1}\left(G_{c}\right)$ by $F(x, \tau)=\bar{\tau} f(x)$. Then

$$
\begin{aligned}
\pi_{c}(F) \eta & =\int_{G} \int_{\mathbb{T}} F(x, \tau) \pi_{c}(x, \tau) \eta \mathrm{d} \tau \mathrm{d} x \\
& =\int_{G} \int_{\mathbb{T}} \bar{\tau} f(x) \bar{\tau} \pi(x) \eta \mathrm{d} \tau \mathrm{d} x=\int_{G} \int_{\mathbb{T}} \bar{\tau}^{2} f(x) \pi(x) \eta \mathrm{d} \tau \mathrm{d} x=0,
\end{aligned}
$$

for all $\eta \in \mathcal{H}$, even though $F$ is not the zero function.

Remark 2.5 Note that if $G$ is nondiscrete we may always extend a representation $\pi: L^{1}(G, c) \rightarrow \mathbb{B}(\mathcal{H})$ to its minimal unitization $L^{1}(G, c)^{\sim}$ by forcing the induced representation, also denoted $\pi$, to satisfy $\pi\left(1_{L^{1}(G, c)^{\sim}}\right)=\operatorname{Id}_{\mathcal{H}}$. Indeed we will need to do this in the sequel. If $L^{1}(G, c)$ is already unital it will always be implied that $\pi\left(1_{L^{1}(G, c)}\right)=\operatorname{Id}_{\mathcal{H}}$.

\subsection{Symmetric Group Algebras and C*-Uniqueness}

Two concepts that will be of great importance when proving our main result Theorem 3.1 are that of symmetric convolution algebras and $C^{*}$-uniqueness.

In the sequel, if $\mathcal{A}$ is a $*$-algebra and $a \in \mathcal{A}$, we let $\sigma_{\mathcal{A}}(a)$ denote the spectrum of $a$ in the algebra $\mathcal{A}$. 
Definition 2.6 A Banach $*$-algebra $\mathcal{A}$ is called symmetric if for all $a \in \mathcal{A}$ we have $\sigma_{\mathcal{A}}\left(a^{*} a\right) \subseteq[0, \infty)$. We will say that a locally compact group $G$ is symmetric if $L^{1}(G)$ is a symmetric Banach $*$-algebra.

Remark 2.7 By the famous Shirali-Ford theorem a Banach $*$-algebra $\mathcal{A}$ is symmetric if and only if it is hermitian (i.e. $a=a^{*} \in \mathcal{A}$ implies $\sigma_{\mathcal{A}}(a) \subset \mathbb{R}$ ).

Note that $C^{*}$-algebras are symmetric [47, Theorem 2.2.5]. Moreover, if $\mathcal{A}$ is a nonunital Banach $*$-algebra, $\mathcal{A}$ is symmetric if and only if its minimal unitization $\tilde{\mathcal{A}}$ is symmetric [50, Theorem (4.7.9)].

Locally compact groups $G$ yielding symmetric (untwisted) convolution algebras $L^{1}(G)$ are of importance due to the following result shown in [30, Theorem 2.8] (though noted several times earlier). Note that we can omit the condition that $G$ should be amenable, as it was recently shown that if $L^{1}(G)$ is symmetric, then $G$ is amenable [52, Corollary 4.8].

Proposition 2.8 If $G$ is a locally compact group the following statements are equivalent.

(i) $L^{1}(G)$ is symmetric.

(ii) $\sigma_{L^{1}(G)}(f)=\sigma_{\mathbb{B}\left(L^{2}(G)\right)}\left(L_{f}\right)$ for all self-adjoint $f \in L^{1}(G)$.

Note that for a locally compact group $G$ and a continuous 2-cocycle $c$ for $G$, the Mackey group $G_{c}$ is amenable if and only if $G$ is amenable [48, Proposition 1.13].

Like in [30], the proofs of some crucial steps will rely on the following result of Hulanicki, see [32], and the extension by Barnes, see [4]. For $a \in \mathcal{A}$, let $\rho_{\mathcal{A}}(a)$ denote the spectral radius of $a$ in $\mathcal{A}$.

Proposition 2.9 Let $\mathcal{A}$ be a $*$-subalgebra of a Banach $*$-algebra $\mathcal{B}$, and suppose there is a faithful $*$-representation $\pi: \mathcal{B} \rightarrow \mathbb{B}(\mathcal{H})$, where $\mathcal{H}$ is a Hilbert space. If $\mathcal{B}$ is unital with unit $1_{\mathcal{B}}$ we require $\pi\left(1_{\mathcal{B}}\right)=\operatorname{Id}_{\mathcal{H}}$. If for all self-adjoint $a \in \mathcal{A}$ we have $\|\pi(a)\|_{\mathbb{B}(\mathcal{H})}=\rho_{\mathcal{A}}(a)$, then

$$
\sigma_{\mathcal{B}}\left(a^{\prime}\right)=\sigma_{\mathbb{B}(\mathcal{H})}\left(\pi\left(a^{\prime}\right)\right)
$$

for all $a^{\prime} \in \mathcal{A}$.

Recall that for an element $b$ in a Banach $*$-algebra $\mathcal{B}$, the spectral radius can be expressed as $\rho_{\mathcal{B}}(b)=\lim _{n \rightarrow \infty}\left\|b^{n}\right\|_{\mathcal{B}}^{1 / n}$ [47, Theorem 1.2.7].

Locally compact groups yielding symmetric convolution algebras have been studied quite extensively. As examples we mention that all locally compact compactly generated groups of polynomial growth yield symmetric convolution algebras [41], as do all compact extensions of nilpotent groups [42, p. 191]. The latter fact will come into play in Sect. 4. Note also that if a group $G$ is locally compact and compactly generated of polynomial growth, so is its Mackey extension $G_{c}$ for any continuous 2-cocycle $c$.

To deduce spectral invariance of $L^{1}(G, c)$ in Theorem 3.1 the strategy in Sect. 3 will be to use Proposition 2.9. In order to do this, we will need a certain norm equality in order for the conditions of Proposition 2.9 to be satisfied. We will restrict to a class of groups for which this is automatic. 
Definition 2.10 Let $\mathcal{B}$ be a Banach $*$-algebra admitting a faithful $*$-representation. We say $\mathcal{B}$ is $C^{*}$-unique if the maximal $C^{*}$-norm $\|\cdot\|_{*}$ given by

$$
\|b\|_{*}=\sup \left\{\|\pi(b)\|_{\mathbb{B}(\mathcal{H})} \mid \pi: \mathcal{B} \rightarrow \mathbb{B}(\mathcal{H}) \text { is a } * \text {-representation of } \mathcal{B}\right\}
$$

for $b \in \mathcal{B}$, is the unique $C^{*}$-norm on $\mathcal{B}$.

We say a locally compact group $G$ is $C^{*}$-unique if $L^{1}(G)$ is $C^{*}$-unique as a Banach *-algebra.

A $C^{*}$-unique group $G$ is amenable, since $C^{*}$-uniqueness in particular implies that the full and reduced group $C^{*}$-algebras of $G$ coincide. The converse is not true $[7,49]$. There are some known examples of $C^{*}$-unique groups. As examples we mention semidirect products of abelian groups, connected metabelian groups, as well as groups where every compactly generated subgroup is of polynomial growth [7, p. 224]. The latter will also come into play in Sect. 4. Moreover, note that if $G$ is a group where every compactly generated subgroup is of polynomial growth, so is its Mackey group $G_{c}$ for any continuous 2-cocycle $c$.

\section{Spectral Invariance of Twisted Convolution Algebras}

All results below will be stated and proved in terms of left representations, i.e. left projective unitary representations of groups and left $*$-representations of the twisted convolution algebras we treated in Sect. 2. This is only due to left representations being more common in the literature. We note that with proper restatements all results in this section also apply to the case of right representations. Indeed we will need to consider right representations in Sect. 4.

We start by presenting the main theorem of the article, and the rest of the section will mostly be dedicated to its proof. Note that some of the lemmas presented leading up to the proof of the main theorem were proved in [13] in a more abstract way. However, as we are considering $\mathbb{T}$-valued 2-cocycles (as opposed to the more general setting of [13]), we believe the clarity offered by the explicit calculations using the Fourier transform below makes the constructions clearer.

Theorem 3.1 Let $G$ be a locally compact group with a continuous 2-cocycle c.

i) If $L^{1}\left(G_{c}\right)$ is $C^{*}$-unique, so is $L^{1}(G, c)$.

ii) If $L^{1}\left(G_{c}\right)$ is symmetric and $C^{*}$-unique and $\pi: L^{1}(G, c) \rightarrow \mathbb{B}(\mathcal{H})$ is a faithful

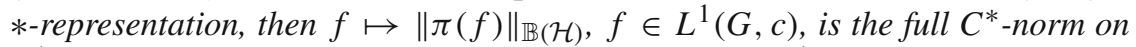
$L^{1}(G, c)$, and $\sigma_{L^{1}(G, c)}(f)=\sigma_{\mathbb{B}(\mathcal{H})}(\pi(f))$ for all $f \in L^{1}(G, c)$.

Remark 3.2 Theorem 3.1 also gives us sufficient conditions for $L^{1}(G, c)$ to be symmetric. Namely, from statement ii) in Theorem 3.1 we see that if $L^{1}\left(G_{c}\right)$ is $C^{*}$-unique and symmetric, then $L^{1}(G, c)$ is spectrally invariant in its (unique) $C^{*}$-completion. Therefore it is spectrally invariant in its enveloping $C^{*}$-algebra, which we know happens if and only if $L^{1}(G, c)$ (and therefore also its minimal unitization if $G$ is nondiscrete) is symmetric [40, p. 340]. 
Remark 3.3 In general $G_{c}$ is less tractable than the group $G$, so at first glance imposing requirements of symmetry and $C^{*}$-uniqueness on $G_{c}$ in Theorem 3.1 might not seem like an improvement. However, untwisted convolution algebras are more tractable than twisted ones, and has been studied to a much larger extent in the literature. In addition, as mentioned in Sect. 2, some classes of symmetric groups and $C^{*}$-unique groups are closed under compact extensions, meaning we for groups $G$ in those classes can impose symmetry and $C^{*}$-uniqueness on $G$ itself rather than on $G_{c}$.

We begin by embedding $L^{p}(G)$ as a subspace of $L^{p}\left(G_{c}\right)$ for $1 \leq p \leq \infty$. Define the $\operatorname{map} j: L^{p}(G) \rightarrow L^{p}\left(G_{c}\right)$ by

$$
j(f)(x, \tau)=\tau f(x) .
$$

Lemma 3.4 Let $G$ be a locally compact group and let c be a continuous 2-cocycle for $G$. Then $j$ defined by (3.1) is an isometric *-homomorphism from $L^{1}(G, c)$ to $L^{1}\left(G_{c}\right)$, and an isometry from $L^{p}(G)$ to $L^{p}\left(G_{c}\right)$ for $1<p \leq \infty$. Moreover, if $f \in L^{1}(G, c)$ and $g \in L^{p}(G)$, we have

$$
j\left(f \bigsqcup_{c} g\right)=j(f) * j(g)
$$

for $p \in[1, \infty]$.

Proof We begin by verifying that $j$ is an isometry for $1 \leq p<\infty$. Let $f \in L^{p}(G)$. Then

$$
\begin{aligned}
\|j(f)\|_{L^{p}\left(G_{c}\right)}^{p} & =\int_{G_{c}}|j(f)(x, \tau)|^{p} \mathrm{~d} \tau \mathrm{d} x=\int_{G} \int_{\mathbb{T}}|\tau f(x)|^{p} \mathrm{~d} \tau \mathrm{d} x \\
& =\int_{G}|f(x)|^{p} \mathrm{~d} x=\|f\|_{L^{p}(G)}^{p} .
\end{aligned}
$$

Likewise, for $p=\infty$ and $f \in L^{\infty}(G)$ we get

$$
\|j(f)\|_{L^{\infty}\left(G_{c}\right)}=\sup _{(x, \tau) \in G_{c}}|j(f)(x, \tau)|=\sup _{(x, \tau) \in G_{c}}|\tau f(x)|=\sup _{x \in G}|f(x)|=\|f\|_{L^{\infty}(G)} .
$$

We now verify that $j$ is a $*$-homomorphism when $p=1$. Let $f_{1}, f_{2} \in L^{1}(G, c)$. Then for all $(x, \tau) \in G_{c}$ we have

$$
\begin{aligned}
\left(j\left(f_{1}\right) * j\left(f_{2}\right)\right)(x, \tau) & =\int_{G_{c}} j\left(f_{1}\right)(y, \xi) j\left(f_{2}\right)\left((y, \xi)^{-1}(x, \tau)\right) \mathrm{d} \xi \mathrm{d} y \\
& =\int_{G} \int_{\mathbb{T}} j\left(f_{1}\right)(y, \xi) j\left(f_{2}\right)\left(y^{-1} x, \bar{\xi} c\left(y, y^{-1}\right) \overline{c\left(y^{-1}, x\right)}\right) \mathrm{d} \xi \mathrm{d} y \\
& =\int_{G} \int_{\mathbb{T}} \xi f_{1}(y) \bar{\xi} \tau c\left(y, y^{-1}\right) \overline{c\left(y^{-1}, x\right)} f_{2}\left(y^{-1} x\right) \mathrm{d} \xi \mathrm{d} y \\
& =\tau \int_{G} f_{1}(y) f_{2}\left(y^{-1} x\right) c\left(y, y^{-1}\right) \overline{c\left(y^{-1}, x\right)} \mathrm{d} y \\
& =\tau \int_{G} f_{1}(y) f_{2}\left(y^{-1} x\right) c\left(y, y^{-1} x\right) \mathrm{d} y \\
& =j\left(f_{1} \natural_{c} f_{2}\right)(x, \tau),
\end{aligned}
$$


where we in the second to last line used (2.3). Doing the same calculation with $f_{2} \in$ $L^{p}(G)$ shows that (3.2) holds.

It then remains to show that $j$ respects the involutions. For $f \in L^{1}(G, c)$ and all $(x, \tau) \in G_{c}$, we have

$$
\begin{aligned}
j(f)^{*}(x, \tau) & =m\left(x^{-1}\right) \overline{j(f)\left((x, \tau)^{-1}\right)}=m\left(x^{-1}\right) \overline{j(f)\left(x^{-1}, \bar{\tau} c\left(x, x^{-1}\right)\right)} \\
& =m\left(x^{-1}\right) \overline{\bar{\tau} c\left(x, x^{-1}\right) f\left(x^{-1}\right)}=m\left(x^{-1}\right) \overline{\tau c\left(x^{-1}, x\right) f\left(x^{-1}\right)} \\
& =\tau f^{*_{c}}(x)=j\left(f^{*_{c}}\right)(x, \tau) .
\end{aligned}
$$

Hence $j(f)^{*}=j\left(f^{*_{c}}\right)$ for all $f \in L^{1}(G, c)$. This finishes the proof.

Since $j$ is an isometry and $L^{p}(G)$ is complete for all $p \in[1, \infty]$, we get that $j\left(L^{p}(G)\right)$ is a closed subspace of $L^{p}\left(G_{c}\right)$. We may actually obtain a quite explicit description of this subspace. To do this, we expand functions in $L^{p}\left(G_{c}\right)$ as Fourier series with respect to their second argument, that is, in the $\mathbb{T}$-variable. Since the measure on $G_{c}$ is the product measure coming from $G$ and $\mathbb{T}$, we have that for any $F \in L^{p}\left(G_{c}\right), 1 \leq p \leq \infty$, and any $x \in G$, the function $\tau \mapsto F(x, \tau)$ is in $L^{p}(\mathbb{T}) \subseteq L^{1}(\mathbb{T})$. Therefore the Fourier coefficients

$$
F_{k}(x)=\int_{\mathbb{T}} F(x, \tau) \bar{\tau}^{k} \mathrm{~d} \tau
$$

are well-defined, and the resulting Fourier series

$$
F(x, \tau)=\sum_{k \in \mathbb{Z}} F_{k}(x) \tau^{k}
$$

converges in $L^{p}(\mathbb{T})$ for $1<p<\infty$. The following lemma then describes the range of $j$.

Lemma 3.5 Let $G$ be a locally compact group and let $c$ be a continuous 2-cocycle for G. For $1 \leq p \leq \infty$ we have $j\left(L^{p}(G)\right)=\left\{F \in L^{p}\left(G_{c}\right) \mid F_{k}=0\right.$ for $\left.k \neq 1\right\}$.

Proof The inclusion $j\left(L^{p}(G)\right) \subseteq\left\{F \in L^{p}\left(G_{c}\right) \mid F_{k}=0\right.$ for $\left.k \neq 1\right\}$ is immediate by (3.1) and (3.3). For the converse containment note that if $F \in\left\{F \in L^{p}\left(G_{c}\right) \mid\right.$ $F_{k}=0$ for $\left.k \neq 1\right\}$, then for all $(x, \tau) \in G_{c}$ we have $F(x, \tau)=\tau F_{1}(x)$. Since the measure on $G_{c}$ is the product measure we must have that $x \mapsto F_{1}(x)$ is in $L^{p}(G)$. Hence $F=j\left(F_{1}\right)$, which proves the lemma.

To simplify notation in the sequel, denote by $L^{1}\left(G_{c}\right)_{n}$ the set

$$
L^{1}\left(G_{c}\right)_{n}:=\left\{F \in L^{1}\left(G_{c}\right) \mid F_{k}=0 \text { for } k \neq n\right\}
$$

It is then immediate that $L^{1}\left(G_{c}\right)_{1}=j\left(L^{1}(G, c)\right)$. We also have the following result. 
Proposition 3.6 Let $G$ be a locally compact group with a continuous 2-cocycle c, let $F \in L^{1}\left(G_{c}\right)$ and let $H \in L^{p}\left(G_{c}\right)$ for some $1 \leq p<\infty$. Then

$$
(F * H)(x, \tau)=\sum_{n \in \mathbb{Z}}\left(F_{n} \natural_{c^{n}} H_{n}\right)(x) \tau^{n},
$$

for all $(x, \tau) \in G_{c}$, where $c^{n}$ is $c$ to the nth power as in Lemma 2.3. Moreover,

$$
\left(F_{n}\right)^{* c^{n}}=\left(F^{*}\right)_{n}
$$

for all $n \in \mathbb{Z}$.

Proof Below we will make use of the Fourier expansions $F(y, \xi)=\sum_{m \in \mathbb{Z}} F_{m}(y) \xi^{m}$ and $H(y, \xi)=\sum_{m \in \mathbb{Z}} H_{m}(y) \xi^{m}$, where $F_{m}$ and $H_{m}$ are obtained through (3.3). We will assume both $F$ and $H$ have finite expansions of the form (3.3). This is sufficient since trigonometric polynomials are dense in $L^{p}(\mathbb{T}), 1 \leq p<\infty$. The extension to the full statement follows by a standard density argument.

Since $\left\{\xi^{m}\right\}_{m \in \mathbb{Z}}$ is an orthonormal system in $L^{2}(\mathbb{T})$, we have for all $(x, \tau) \in G_{c}$

$$
\begin{aligned}
(F * H)(x, \tau) & =\int_{G} \int_{\mathbb{T}} F(y, \xi) H\left((y, \xi)^{-1}(x, \tau)\right) \mathrm{d} \xi \mathrm{d} y \\
& =\int_{G} \int_{\mathbb{T}} F(y, \xi) H\left(y^{-1} x, \bar{\xi} c\left(y^{-1}, y\right) \tau \overline{c\left(y^{-1}, x\right)}\right) \mathrm{d} \xi \mathrm{d} y \\
& =\int_{G} \int_{\mathbb{T}} \sum_{m \in \mathbb{Z}} F_{m}(y) \xi^{m} \cdot \sum_{n \in \mathbb{Z}} H_{n}\left(y^{-1} x\right) \bar{\xi}^{n} \tau^{n}\left(c\left(y, y^{-1} x\right)\right)^{n} \mathrm{~d} \xi \mathrm{d} y \\
& =\int_{G} \sum_{n \in \mathbb{Z}} F_{n}(y) H_{n}\left(y^{-1} x\right) c^{n}\left(y, y^{-1} x\right) \tau^{n} \mathrm{~d} y \\
& =\sum_{n \in \mathbb{Z}}\left(\int_{G} F_{n}(y) H_{n}\left(y^{-1} x\right) c^{n}\left(y, y^{-1} x\right) \mathrm{d} y\right) \tau^{n} \\
& =\sum_{n \in \mathbb{Z}}\left(F_{n} \natural_{c^{n}} H_{n}\right)(x) \tau^{n}
\end{aligned}
$$

where we at the third equality used (2.3). This establishes (3.4).

For any $F \in L^{1}\left(G_{c}\right)$ we also have

$$
\begin{aligned}
\left(F^{*}\right)_{n}(x) & =\int_{\mathbb{T}} F^{*}(x, \tau) \bar{\tau}^{n} \mathrm{~d} \tau \\
& =\int_{\mathbb{T}} m\left(x^{-1}\right) \overline{F\left(x^{-1}, \bar{\tau} c\left(x^{-1}, x\right)\right)} \bar{\tau}^{n} \mathrm{~d} \tau \\
& =m\left(x^{-1}\right) \int_{\mathbb{T}} \overline{F\left(x^{-1}, \tau c\left(x^{-1}, x\right)\right)} \tau^{n} \mathrm{~d} \tau \\
& =m\left(x^{-1}\right) \int_{\mathbb{T}} \overline{F\left(x^{-1}, \tau\right)} \tau^{n} \overline{c\left(x^{-1}, x\right)^{n}} \mathrm{~d} \tau
\end{aligned}
$$




$$
\begin{aligned}
& =m\left(x^{-1}\right) \overline{c\left(x^{-1}, x\right)^{n}} \overline{\int_{\mathbb{T}} F\left(x^{-1}, \tau\right) \bar{\tau}^{n} \mathrm{~d} \tau} \\
& =m\left(x^{-1}\right) \overline{c^{n}\left(x^{-1}, x\right) F_{n}\left(x^{-1}\right)} \\
& =\left(F_{n}\right)^{* c^{n}}(x)
\end{aligned}
$$

for all $x \in G$, which establishes (3.5).

The following corollary is then immediate.

Corollary 3.7 Let $G$ be a locally compact group and let c be a continuous 2-cocycle for $G$. Then $L^{1}\left(G_{c}\right)_{n} \cong L^{1}\left(G, c^{n}\right)$ as Banach $*$-algebras.

As a final preparation before proving Theorem 3.1, we need the following lemma.

Lemma 3.8 Let $G$ be a locally compact group and let c be a continuous 2-cocycle for $G$. For $f \in L^{1}(G, c)$ we then have

$$
\rho_{L^{1}(G, c)}(f)=\rho_{L^{1}\left(G_{c}\right)}(j(f))
$$

If, in addition, $f$ is self-adjoint we get

$$
\rho_{\mathbb{B}\left(L^{2}(G)\right)}\left(L_{f}^{c}\right)=\rho_{\mathbb{B}\left(L^{2}\left(G_{c}\right)\right)}\left(L_{j(f)}\right) .
$$

Proof Since $j: L^{1}(G, c) \rightarrow L^{1}\left(G_{c}\right)$ is an isometric $*$-homomorphism we have

$$
\rho_{L^{1}(G, c)}(f)=\lim _{n \rightarrow \infty}\left\|f^{n}\right\|_{L^{1}(G, c)}^{1 / n}=\lim _{n \rightarrow \infty}\left\|j(f)^{n}\right\|_{L^{1}\left(G_{c}\right)}^{1 / n}=\rho_{L^{1}\left(G_{c}\right)}(j(f)),
$$

which proves the first statement.

For the second statement, let $f \in L^{1}(G, c)$ be self-adjoint. Since $f$ is self-adjoint and $L_{f}^{c}$ and $L_{j(f)}$ realize $f$ and $j(f)$ as bounded operators on Hilbert spaces, i.e. as elements of a $C^{*}$-algebra, we have

$$
\rho_{\mathbb{B}\left(L^{2}(G)\right)}\left(L_{f}^{c}\right)=\left\|L_{f}^{c}\right\|_{\mathbb{B}\left(L^{2}(G)\right)} \quad \text { and } \quad \rho_{\mathbb{B}\left(L^{2}\left(G_{c}\right)\right)}\left(L_{j(f)}\right)=\left\|L_{j(f)}\right\|_{\mathbb{B}\left(L^{2}\left(G_{c}\right)\right)},
$$

see [47, Theorem 2.1.1]. Hence it suffices to show that $\left\|L_{f}^{c}\right\|_{\mathbb{B}\left(L^{2}(G)\right)}=\left\|L_{j(f)}\right\|_{\mathbb{B}\left(L^{2}\left(G_{c}\right)\right)}$. To do this, note first that by Lemma 3.4

$$
L_{j(f)} j(g)=j(f) * j(g)=j\left(f \natural_{c} g\right)=j\left(L_{f}^{c} g\right)
$$

for any $g \in L^{2}(G)$. Moreover, by Proposition 3.6 we see that $\left.L_{j(f)}\right|_{j\left(L^{2}(G)\right)^{\perp}}=0$. Since $j: L^{2}(G) \rightarrow L^{2}\left(G_{c}\right)$ is an isometry it then follows that $\left\|L_{f}^{c}\right\|_{\mathbb{B}\left(L^{2}(G)\right)}=$ $\left\|L_{j(f)}\right\|_{\mathbb{B}\left(L^{2}\left(G_{c}\right)\right)}$, which finishes the proof.

We are finally ready to prove Theorem 3.1. 
Proof of Theorem 3.1 We begin by proving i). Let $\pi: L^{1}(G, c) \rightarrow \mathbb{B}(\mathcal{H})$ be a faithful *-representation. As $G_{c}$ is assumed $C^{*}$-unique, $G_{c}$ is in particular amenable, so it follows that $G$ is also amenable. Then Proposition 2.4 gives that $f \mapsto\left\|L_{f}^{c}\right\|_{\mathbb{B}\left(L^{2}(G)\right)}$, $f \in L^{1}(G, c)$, is the maximal $C^{*}$-norm on $L^{1}(G, c)$. Hence it suffices to prove that $\|\pi(f)\|_{\mathbb{B}(\mathcal{H})}=\left\|L_{f}^{c}\right\|_{\mathbb{B}\left(L^{2}(G)\right)}$ for all $f \in L^{1}(G, c)$. To do this, we will first extend $\pi$ to a faithful $*$-representation of $L^{1}\left(G_{c}\right)$. The obvious attempt at a $*$-representation of $L^{1}\left(G_{c}\right)$, namely the integrated representation of $\pi_{c}: G_{c} \rightarrow \mathcal{U}(\mathcal{H})$ as in $(2.5)$, is in general not faithful as noted at the end of Sect. 2.1. The construction of the desired faithful $*$-representation $\tilde{\pi}$ of $L^{1}\left(G_{c}\right)$ is therefore more involved.

For all $n \in \mathbb{Z}$ we know by Corollary 3.7 that $L^{1}\left(G_{c}\right)_{n} \cong L^{1}\left(G, c^{n}\right)$ as Banach $*$-algebras, and in the sequel we make this identification to ease notation. For any $n \in \mathbb{Z} \backslash\{1\}$ we define

$$
\pi^{(n)}:=L^{c^{n}}: L^{1}\left(G, c^{n}\right) \rightarrow \mathbb{B}\left(L^{2}(G)\right),
$$

and set

$$
\pi^{(1)}:=\pi: L^{1}(G, c) \rightarrow \mathbb{B}(\mathcal{H}) .
$$

Then $\pi^{(n)}$ is a faithful $*$-representation of $L^{1}\left(G, c^{n}\right)$ for all $n \in \mathbb{Z}$. Moreover, we set

$$
\mathcal{H}^{(n)}= \begin{cases}L^{2}(G) & \text { if } n \in \mathbb{Z} \backslash\{1\} \\ \mathcal{H} & \text { if } n=1\end{cases}
$$

Note that $\oplus_{k \in \mathbb{Z}} \mathbb{B}\left(\mathcal{H}^{(k)}\right)$ becomes a $C^{*}$-algebra by realizing $\oplus_{k \in \mathbb{Z}} \mathbb{B}\left(\mathcal{H}^{(k)}\right) \quad \subset$ $\mathbb{B}\left(\oplus_{k \in \mathbb{Z}} \mathcal{H}^{(k)}\right)$, where $\oplus_{k \in \mathbb{Z}} \mathcal{H}^{(k)}$ is the Hilbert direct sum. We then consider the map $\tilde{\pi}: L^{1}\left(G_{c}\right) \rightarrow \oplus_{k \in \mathbb{Z}} \mathbb{B}\left(\mathcal{H}^{(k)}\right)$ which for $F \in L^{1}\left(G_{c}\right)$ is given by

$$
F \mapsto\left(F_{k}\right)_{k \in \mathbb{Z}} \mapsto \bigoplus_{k \in \mathbb{Z}} \pi^{(k)}\left(F_{k}\right)
$$

We must verify that this is a faithful $*$-homomorphism. Continuity will then follow since any $*$-homomorphism from a Banach $*$-algebra to a $C^{*}$-algebra is continuous [47, Theorem 2.1.7]

For $F, H \in L^{1}\left(G_{c}\right)$ it then follows from (3.4) that

$$
\tilde{\pi}(F * H)=\bigoplus_{k \in \mathbb{Z}} \pi^{(k)}\left(F_{k} \natural_{c^{k}} H_{k}\right)=\bigoplus_{k \in \mathbb{Z}} \pi^{(k)}\left(F_{k}\right) \circ \pi^{(k)}\left(H_{k}\right)=\tilde{\pi}(F) \tilde{\pi}(H) .
$$

It also follows from (3.5) that

$$
\tilde{\pi}\left(F^{*}\right)=\bigoplus_{k \in \mathbb{Z}} \pi^{(k)}\left(\left(F^{*}\right)_{k}\right)=\bigoplus_{k \in \mathbb{Z}} \pi^{(k)}\left(\left(F_{k}\right)^{*} c^{k}\right)=\bigoplus_{k \in \mathbb{Z}} \pi^{(k)}\left(F_{k}\right)^{*}=\tilde{\pi}(F)^{*} .
$$

We conclude that $\tilde{\pi}$ is a continuous $*$-homomorphism.

Now suppose $F \in L^{1}\left(G_{c}\right)$ is such that $\tilde{\pi}(F)=0$. Then $\pi^{(k)}\left(F_{k}\right)=0$ for all $k \in \mathbb{Z}$, and since $\pi^{(k)}: L^{1}\left(G, c^{k}\right) \rightarrow \mathbb{B}\left(\mathcal{H}^{(k)}\right)$ are all faithful, we conclude that $F_{k}=0$ for 
all $k \in \mathbb{Z}$. Since the Fourier transform is injective on $L^{1}$, this happens if and only if $F=0$ almost everywhere, i.e. if $F=0$ in $L^{1}\left(G_{c}\right)$. We deduce that $\tilde{\pi}$ is a faithful *-homomorphism.

Observe that since $\mathcal{H}^{(1)}=\mathcal{H}$, the two representations $\pi: L^{1}(G, c) \rightarrow \mathbb{B}(\mathcal{H})$ and $\tilde{\pi} \circ j: L^{1}(G, c) \rightarrow \mathbb{B}\left(\mathcal{H}^{(1)}\right)$ can naturally be identified. Using the $C^{*}$-identity, $C^{*}$-uniqueness of $G_{c}$, and Lemma 3.8 we then obtain

$$
\begin{aligned}
\|\pi(f)\|_{\mathbb{B}(\mathcal{H})}^{2} & =\left\|\pi\left(f^{*} \natural_{c} f\right)\right\|_{\mathbb{B}(\mathcal{H})}=\left\|\tilde{\pi}\left(j\left(f^{*} \natural_{c} f\right)\right)\right\|_{\oplus k \in \mathbb{Z} \mathbb{B}\left(\mathcal{H}^{(k)}\right)} \\
& =\left\|L_{j\left(f^{*} \natural_{c} f\right)}\right\|_{\mathbb{B}\left(L^{2}\left(G_{c}\right)\right)}=\left\|L_{f^{*} \natural_{c} f}^{c}\right\|_{\mathbb{B}\left(L^{2}(G)\right)}=\left\|L_{f}^{c}\right\|_{\mathbb{B}\left(L^{2}(G)\right)}^{2}
\end{aligned}
$$

for all $f \in L^{1}(G, c)$, which proves i).

To prove ii), let $f \in L^{1}(G, c)$ be self-adjoint. Using Lemma 3.8, Proposition 2.8 and i) of Theorem 3.1, we have the following chain of equalities

$$
\begin{aligned}
\rho_{L^{1}(G, c)}(f) & =\rho_{L^{1}\left(G_{c}\right)}(j(f))=\rho_{\mathbb{B}\left(L^{2}\left(G_{c}\right)\right)}\left(L_{j(f)}\right) \\
& =\rho_{\mathbb{B}\left(L^{2}(G)\right)}\left(L_{f}^{c}\right)=\left\|L_{f}^{c}\right\|_{\mathbb{B}\left(L^{2}(G)\right)}=\|\pi(f)\|_{\mathbb{B}(\mathcal{H}) .} .
\end{aligned}
$$

By Proposition 2.9 it then follows that $\sigma_{L^{1}(G, c)}(f)=\sigma_{\mathbb{B}(\mathcal{H})}(\pi(f))$ for all $f \in$ $L^{1}(G, c)$.

Remark 3.9 Looking at the proof of Theorem 3.1 we might hope in light of results on symmetric (Banach) $*$-algebras in e.g. $[6,19,20,29,45]$ that it is possible to obtain similar results for the algebras considered in these papers. However, considering the crucial role $C^{*}$-uniqueness plays in order to get spectral invariance for all $*$-representations for the $*$-algebra in Theorem 3.1, it would look like a key ingredient in a proof of such a result should be analogous $C^{*}$-uniqueness results for these algebras, and for the time being these remain elusive.

\section{Applications to Gabor Analysis}

We begin by introducing the central concepts of Gabor analysis, before formulating the main result of this section. We then rephrase the setting of the problem in terms spectral invariance of a certain convolution algebra and use Theorem 3.1 to prove the result.

Throughout this section $G$ will be a locally compact abelian group and $\widehat{G}$ will be its Pontryagin dual. Note that we will write the group operation additively. Moreover, $\Delta$ will denote a closed cocompact subgroup of the time-frequency plane $G \times \widehat{G}$. The reason for restricting to cocompact subgroups will be made clear in Remark 4.1. We fix a Haar measure on $G$ and equip $\widehat{G}$ with the dual measure such that Plancherel's formula holds [12, Theorem 3.4.8]. We also fix a Haar measure on $\Delta$ (which in the sequel will be denoted $\mathrm{d} z$ ), and give $(G \times \widehat{G}) / \Delta$ the unique measure such that Weil's formula holds [34, equation (2.4)]. The size of $\Delta$ is the quantity $s(\Delta):=\mu((G \times \widehat{G}) / \Delta)$, where $\mu$ is the chosen Haar measure. As $\Delta$ is cocompact in $G \times \widehat{G}$, we have $s(\Delta)<\infty$. 
We proceed to introduce the two unitary operators most relevant for Gabor analysis. Given $x \in G$ and $\omega \in \widehat{G}$, we define the translation operator $T_{x}$ and modulation operator $M_{\omega}$ on $L^{2}(G)$ by

$$
\left(T_{x} f\right)(t)=f(t-x), \quad\left(M_{\omega} f\right)(t)=\omega(t) f(t)
$$

for $f \in L^{2}(G)$ and $t \in G$. Moreover, we define a time-frequency shift by

$$
\pi(x, \omega):=M_{\omega} T_{x}
$$

for $x \in G$ and $\omega \in \widehat{G}$.

Having introduced both translation and modulation we may define the subgroup of $G \times \widehat{G}$ which will be of greatest importance to us when proving Theorem 4.2. This is due to the reformulations of the frame operator in (4.6) and (4.8) below. The adjoint subgroup of $\Delta$, denoted $\Delta^{\circ}$, is the closed subgroup of $G \times \widehat{G}$ defined by

$$
\Delta^{\circ}:=\{w \in G \times \widehat{G} \mid \pi(z) \pi(w)=\pi(w) \pi(z) \text { for all } z \in \Delta\}
$$

Its importance for time-frequency analysis was first realized in [17]. We may identify $\Delta^{\circ}$ with $\left((G \times \widehat{G}) / \Delta \widehat{)}\right.$ as in $\left[34\right.$, p. 234] to pick the dual measure on $\Delta^{\circ}$ corresponding to the measure on $(G \times \widehat{G}) / \Delta . \Delta$ is cocompact in $G \times \widehat{G}$, so $\Delta^{\circ}$ is discrete. The induced measure on $\Delta^{\circ}$ is the counting measure scaled with the constant $s(\Delta)^{-1}$ [35, equation (13)].

Given $g \in L^{2}(G)$, the Gabor system over $\Delta$ with generator $g$ is a family $\mathcal{G}(g ; \Delta):=$ $(\pi(z) g)_{z \in \Delta}$. It is called a Gabor frame if it is a (continuous) frame for $L^{2}(G)[1,34,36]$ in the sense that the following conditions are satisfied:

i) The family $\mathcal{G}(g, \Delta)$ is weakly measurable, i.e. for every $f \in L^{2}(G)$ the map $z \mapsto\langle f, \pi(z) g\rangle$ is measurable.

ii) There exist positive constants $C, D>0$ such that for all $f \in L^{2}(G)$ we have that

$$
C\|f\|_{2}^{2} \leq \int_{\Delta}|\langle f, \pi(z) g\rangle|^{2} \mathrm{~d} z \leq D\|f\|_{2}^{2}
$$

Remark 4.1 Gabor frames $\mathcal{G}(g ; \Delta)$ for $L^{2}(G)$ with $g \in L^{2}(G)$ can only exist if $\Delta$ is cocompact [34, Theorem 5.1]. Indeed, this is also the case if we consider finitely many functions $g_{1}, \ldots, g_{k} \in L^{2}(G)$ and a Gabor system $\mathcal{G}\left(g_{1}, \ldots, g_{k} ; \Delta\right)$ as in Remark 4.5 below [35, Lemma 4.9]. The same is true if we consider matrix frames introduced in [3], see [3, Proposition 4.29].

If $\mathcal{G}(g ; \Delta)$ is weakly measurable and $D<\infty$ for this family, we say $\mathcal{G}(g ; \Delta)$ is a Bessel system. Associated to any Bessel system $\mathcal{G}(g ; \Delta)$ is a linear bounded operator known as the frame operator associated to $\mathcal{G}(g ; \Delta)$. It is the operator

$$
\begin{aligned}
S: L^{2}(G) & \rightarrow L^{2}(G) \\
f & \mapsto \int_{\Delta}\langle f, \pi(z) g\rangle \pi(z) g \mathrm{~d} z,
\end{aligned}
$$


where we interpret the integral weakly. It is well known in frame theory that $S$ commutes with all time-frequency shifts $\pi(z)$ when $z \in \Delta$, and that $\mathcal{G}(g ; \Delta)$ is a Gabor frame for $L^{2}(G)$ if and only if $S$ is invertible on $L^{2}(G)$. Moreover, it is not hard to see that $S$ is a positive operator.

Now let $\mathcal{G}(g ; \Delta)$ be a Gabor frame for $L^{2}(G)$. Using that the frame operator commutes with time-frequency shifts from $\Delta$, we have

$$
f=S^{-1} S f=\int_{\Delta}\langle f, \pi(z) g\rangle \pi(z) S^{-1} g \mathrm{~d} z
$$

for all $f \in L^{2}(G)$. The function $S^{-1} g$ is known as the canonical dual atom of $g$. Moreover, we have

$$
f=S^{-1 / 2} S S^{-1 / 2} f=\int_{\Delta}\left\langle f, \pi(z) S^{-1 / 2} g\right\rangle \pi(z) S^{-1 / 2} g \mathrm{~d} z
$$

for all $f \in L^{2}(G)$. The function $S^{-1 / 2} g$ is known as the canonical tight atom associated to $g$.

As a last preparation before presenting the main result of this section we must introduce a function space. Let $g \in L^{2}(G)$. We define the short-time Fourier transform with respect to $g$ to be the operator $V_{g}: L^{2}(G) \rightarrow L^{2}(G \times \widehat{G})$ given by

$$
V_{g} f(z)=\langle f, \pi(z) g\rangle
$$

for $f \in L^{2}(G)$ and $z \in G \times \widehat{G}$. Using this, we define the Feichtinger algebra $S_{0}(G)$ by

$$
S_{0}(G):=\left\{f \in L^{2}(G) \mid V_{f} f \in L^{1}(G \times \widehat{G})\right\} .
$$

The Feichtinger algebra is known as a nice space of test functions for time-frequency analysis, and its elements have good decay in both time and frequency. We refer the reader to [33] for more information on the Feichtinger algebra. At last, we may state the main theorem of this section.

Theorem 4.2 Let $\Delta \subseteq G \times \widehat{G}$ be a closed cocompact subgroup, and suppose $g \in$ $S_{0}(G)$ is such that $\mathcal{G}(\bar{g} ; \Delta)$ is a Gabor frame for $L^{2}(G)$. Then $S^{-1} g, S^{-1 / 2} g \in S_{0}(G)$ as well.

In the case when $\Delta$ is a separable lattice in $\mathbb{R}^{d} \times \widehat{\mathbb{R}^{d}}$, Theorem 4.2 was proved in [30], and it was claimed to hold for general lattices in phase spaces of locally compact abelian groups. It is possible that their techniques can be adapted to the setting of closed cocompact subgroups. However, it will turn out that the result is easier to deduce by using Theorem 3.1, thereby circumventing any need to use the periodization techniques of [30]. In order to show Theorem 4.2 we will reformulate the above setup to incorporate twisted convolution algebras. As a first step towards this we present the Fundamental Identity of Gabor Analysis. We refer the reader to [51, Proposition 2.11] for a proof. There the Schwartz-Bruhat space is used, but the proof can easily be adapted to the case of $S_{0}(G)$. For the case of modulation spaces, see for example [18] or [25] 
Proposition 4.3 Let $f, g, h \in S_{0}(G)$. Then

$$
\int_{\Delta}\langle f, \pi(z) g\rangle \pi(z) h \mathrm{~d} z=\frac{1}{s(\Delta)} \sum_{w \in \Delta^{\circ}}\langle\pi(w) h, g\rangle \pi(w)^{*} f
$$

where we interpret the integral and the sum weakly in $L^{2}(G)$.

Proposition 4.3 allows us to rewrite the frame operator $S$ for $\mathcal{G}(g ; \Delta)$ as

$$
S f=\int_{\Delta}\langle f, \pi(z) g\rangle \pi(z) g \mathrm{~d} z=\frac{1}{s(\Delta)} \sum_{w \in \Delta^{\circ}}\langle\pi(w) g, g\rangle \pi(w)^{*} f .
$$

This observation is key in rephrasing the problem. It is the right hand side which is of importance to us, and it will be most natural to restate the frame operator in terms of a right $*$-representation of a twisted convolution algebra, see Equation (4.8).

We will also need the continuous 2-cocycle on $G \times \widehat{G}$ known as the Heisenberg 2-cocycle [51, p. 263]. It is the map $c:(G \times \widehat{G}) \times(G \times \widehat{G}) \rightarrow \mathbb{T}$ given by

$$
c((x, \omega),(y, \tau))=\overline{\tau(x)}
$$

for $(x, \omega),(y, \tau) \in G \times \widehat{G}$.

Remark 4.4 Having introduced the Heisenberg 2-cocycle $c$ we may also describe $\Delta^{\circ}$ without using time-frequency shifts directly by ways of

$$
\Delta^{\circ}=\{w \in G \times \widehat{G} \mid c(w, z) \bar{c}(z, w)=1 \text { for all } z \in \Delta\}
$$

Restricting to $\Delta^{\circ}$, we construct the twisted convolution algebra $\ell^{1}\left(\Delta^{\circ}, \bar{c}\right)$ as in Sect. 2. Now the map

$$
\begin{aligned}
\pi^{*}: G \times \widehat{G} & \rightarrow \mathcal{U}\left(L^{2}(G)\right) \\
(x, \omega) & \mapsto \pi(x, \omega)^{*}
\end{aligned}
$$

defines a right $\bar{c}$-projective unitary representation. A right projective unitary representation of a group may also be viewed as a (left) projective unitary representation of its opposite group. We also get a right $\bar{c}$-projective unitary representation of $\Delta^{\circ}$, which we also denote by $\pi^{*}$. The integrated representation defines a right $*$-representation $\pi^{*}: \ell^{1}\left(\Delta^{\circ}, \bar{c}\right) \rightarrow \mathbb{B}\left(L^{2}(G)\right)$. This representation leaves $S_{0}(G)$ invariant, i.e. $\pi^{*}\left(\ell^{1}\left(\Delta^{\circ}, \bar{c}\right)\right) S_{0}(G) \subseteq S_{0}(G)$ [35, Theorem 3.4]. Given $a=\left(a_{w}\right)_{w \in \Delta^{\circ}} \in \ell^{1}\left(\Delta^{\circ}, \bar{c}\right)$ and $f \in L^{2}(G)$ we have

$$
\pi^{*}(a) f=\frac{1}{s(\Delta)} \sum_{w \in \Delta^{\circ}} a_{w} \pi(w)^{*} f
$$

Also, this $*$-representation is known to be faithful [51, Proposition 2.2]. Moreover, for $g \in S_{0}(G)$ we have $(\langle\pi(w) g, g\rangle)_{w \in \Delta^{\circ}} \in \ell^{1}\left(\Delta^{\circ}, \bar{c}\right)$ [35, Theorem 3.4]. Using (4.6) for the Gabor system $\mathcal{G}(g ; \Delta), g \in S_{0}(G)$, we now see that 


$$
S f=\pi^{*}\left((\langle\pi(w) g, g\rangle)_{w \in \Delta^{\circ}}\right) f
$$

for $f \in L^{2}(G)$. We are finally ready to prove Theorem 4.2. The proof follows the same general outline as the proof of [30, Theorem 4.2], but without use of periodization techniques unique to the Gabor analysis setting.

Proof of Theorem 4.2 If $g \in S_{0}(G)$ is such that $\mathcal{G}(g ; \Delta)$ is a Gabor frame for $L^{2}(G)$, then the corresponding frame operator $S$ is invertible. By (4.8) we may write $S f=\pi^{*}\left((\langle\pi(w) g, g\rangle)_{w \in \Delta^{\circ}}\right) f$ for any $f \in L^{2}(G)$. Since $\Delta^{\circ}$ is abelian, every compactly generated subgroup of $\Delta^{\circ}$ is of polynomial growth by the structure theorem for compactly generated locally compact abelian groups [12, Theorem 4.2.2]. Hence every compactly generated subgroup of $\Delta_{\bar{c}}^{\circ}$ is also of polynomial growth, since it is a compact extension of $\Delta^{\circ}$. It follows that $\Delta_{\bar{c}}^{\circ}$ is $C^{*}$-unique. Moreover, $\Delta_{\bar{c}}^{\circ}$ is nilpotent of class 1 as $\Delta^{\circ}$ is abelian, so it follows that $\ell^{1}\left(\Delta_{\bar{c}}^{\circ}\right)$ is symmetric. By Theorem 3.1 we then have that $\ell^{1}\left(\Delta^{\circ}, \bar{c}\right)$ is spectrally invariant in $\mathbb{B}\left(L^{2}(G)\right)$. Hence there is $a=\left(a_{w}\right)_{w \in \Delta^{\circ}} \in$ $\ell^{1}\left(\Delta^{\circ}, \bar{c}\right)$ such that $a \natural_{\bar{c}}(\langle\pi(w) g, g\rangle)_{w \in \Delta^{\circ}}=1_{\ell^{1}\left(\Delta^{\circ}, \bar{c}\right)}=(\langle\pi(w) g, g\rangle)_{w \in \Delta^{\circ}} \natural_{\bar{c}} a$ and

$$
S^{-1} f=\pi^{*}(a) f
$$

for all $f \in L^{2}(G)$. Since $\pi^{*}\left(\ell^{1}\left(\Delta^{\circ}, \bar{c}\right)\right)$ leaves $S_{0}(G)$ invariant, it follows that $S^{-1} g \in$ $S_{0}(G)$.

Since $S$, hence also $S^{-1}$, is a positive operator, we may also take the square root of the image of $a$ under $\pi^{*}$ in $\mathbb{B}\left(L^{2}(G)\right)$. By spectral invariance and the fact that Banach *-algebras are closed under holomorphic functional calculus [11, p. 212] it follows that there is $b=\left(b_{w}\right)_{w \in \Delta^{\circ}} \in \ell^{1}\left(\Delta^{\circ}, \bar{c}\right)$ such that

$$
S^{-1 / 2} f=\pi^{*}(b) f
$$

for all $f \in L^{2}(G)$. Once again, since $\pi^{*}\left(\ell^{1}\left(\Delta^{\circ}, \bar{c}\right)\right)$ leaves $S_{0}(G)$ invariant, it follows that $S^{-1 / 2} g \in S_{0}(G)$. This finishes the proof.

Remark 4.5 There are no issues extending this to multi-window Gabor frames, i.e. the case of $g_{1}, \ldots, g_{k} \in S_{0}(G)$ such that $\mathcal{G}\left(g_{1}, \ldots, g_{k} ; \Delta\right):=\mathcal{G}\left(g_{1} ; \Delta\right) \cup \cdots \cup \mathcal{G}\left(g_{k} ; \Delta\right)$ is a frame for $L^{2}(G)$. Indeed, the only real difference is that we in (4.8) will need to consider $\pi^{*}\left(\left(\sum_{i=1}^{k}\left\langle\pi(w) g_{i}, g_{i}\right\rangle\right)_{w \in \Delta^{\circ}}\right)$. This is of no real consequence for the proofs. Hence we may conclude that for a multi-window Gabor frame $\mathcal{G}\left(g_{1}, \ldots, g_{k} ; \Delta\right)$ for $L^{2}(G)$ with $g_{1}, \ldots, g_{k} \in S_{0}(G)$ and associated (multi-window) frame operator $S$ we get $S^{-1} g_{1}, \ldots, S^{-1} g_{k}, S^{-1 / 2} g_{1}, \ldots, S^{-1 / 2} g_{k} \in S_{0}(G)$. Indeed one can go even further and do this for the matrix Gabor frames introduced in [3], which generalize multi-window super Gabor frames, using the setup from the same article. The key observation for doing this is that since $\ell^{1}\left(\Delta^{\circ}, \bar{c}\right)$ is spectrally invariant in $\mathbb{B}\left(L^{2}(G)\right)$ we also have that $M_{n}\left(\ell^{1}\left(\Delta^{\circ}, \bar{c}\right)\right)$ is spectrally invariant in $M_{n}\left(\mathbb{B}\left(L^{2}(G)\right)\right)$ for any $n \in \mathbb{N}[53]$.

Acknowledgements The author would like to thank Michael Leinert for valuable feedback on an earlier draft of the article. Moreover, the author would like to thank Eduard Ortega for pointing out the concept of $C^{*}$-uniqueness, which broadened the scope and application of the article significantly. Lastly, the author 
also wishes to thank Eirik Berge, Mads S. Jakobsen, Franz Luef, and Eirik Skrettingland for valuable discussions during the development of the article.

Funding Open access funding provided by NTNU Norwegian University of Science and Technology (incl St. Olavs Hospital - Trondheim University Hospital).

Open Access This article is licensed under a Creative Commons Attribution 4.0 International License, which permits use, sharing, adaptation, distribution and reproduction in any medium or format, as long as you give appropriate credit to the original author(s) and the source, provide a link to the Creative Commons licence, and indicate if changes were made. The images or other third party material in this article are included in the article's Creative Commons licence, unless indicated otherwise in a credit line to the material. If material is not included in the article's Creative Commons licence and your intended use is not permitted by statutory regulation or exceeds the permitted use, you will need to obtain permission directly from the copyright holder. To view a copy of this licence, visit http://creativecommons.org/licenses/by/4.0/.

\section{References}

1. Ali, S.T., Antoine, J.-P., Gazeau, J.-P.: Continuous frames in Hilbert space. Ann. Phys. 222(1), 1-37 (1993)

2. Austad, A., Enstad, U.: Heisenberg modules as function spaces. J. Fourier Anal. Appl., 26(2), (2020)

3. Austad, A., Jakobsen, M. S., Luef, F.: Gabor duality theory for Morita equivalent $C^{*}$-algebras. )Int. J. Math. 31(10), 2050073, 34, (2020)

4. Barnes, B.A.: When is the spectrum of a convolution operator on $L^{p}$ independent of $p$ ? Proc. Edinb. Math. Soc. (2) 33(2), 327-332 (1990)

5. Baskakov, A.G.: Wiener's theorem and asymptotic estimates for elements of inverse matrices. Funktsional. Anal. i Prilozhen. 24(3), 64-65 (1990)

6. Beltiţă, I., Beltiţă, D.: Inverse-closed algebras of integral operators on locally compact groups. Ann. Henri Poincaré 16(5), 1283-1306 (2015)

7. Boidol, J.: Group algebras with a unique $C^{*}$-norm. J. Funct. Anal. 56(2), 220-232 (1984)

8. Christensen, O.: Atomic decomposition via projective group representations. Rocky Mt. J. Math. 26(4), 1289-1312 (1996)

9. Connes, A.: $C^{*}$-algèbres et géométrie différentielle. C. R. Acad. Sci. Paris Sér. A-B, 290(13):A599A604 (1980)

10. Connes, A.: Noncommutative Geometry. Academic Press Inc., New York (1994)

11. Dales, H.G.: Banach Algebras and Automatic Continuity, volume 24 of London Mathematical Society Monographs. New Series. The Clarendon Press, Oxford University Press, New York, (2000). Oxford Science Publications

12. Deitmar, A., Echterhoff, S.: Principles of Harmonic Analysis, 2nd edn. Universitext. Springer, Cham (2014)

13. Edwards, C.M., Lewis, J. T.: Twisted group algebras. I, II. Comm. Math. Phys., 13:119-130; ibid. 131-141, (1969)

14. Enstad, U.: The Balian-Low theorem for locally compact abelian groups and vector bundles. J. Math. Pures Appl. 9(139), 143-176 (2020)

15. Feichtinger, H.G., Gröchenig, K.H.: Banach spaces related to integrable group representations and their atomic decompositions. I. J. Funct. Anal. 86(2), 307-340 (1989)

16. Feichtinger, H.G., Gröchenig, K.H.: Banach spaces related to integrable group representations and their atomic decompositions. II. Monatsh. Math. 108(2-3), 129-148 (1989)

17. Feichtinger, H.G., Kozek, W.: Quantization of TF lattice-invariant operators on elementary LCA groups. In: Gabor Analysis and Algorithms, Appl. Numer. Harmon. Anal., pp. 233-266. Birkhäuser Boston, Boston, MA, (1998)

18. Feichtinger, H.G., Luef, F.: Wiener amalgam spaces for the fundamental identity of Gabor analysis. Collect. Math., (Vol. Extra):233-253 (2006)

19. Fendler, G., Gröchenig, K., Leinert, M.: Symmetry of weighted $L^{1}$-algebras and the GRS-condition. Bull. Lond. Math. Soc. 38(4), 625-635 (2006) 
20. Fendler, G., Gröchenig, K., Leinert, M., Ludwig, J., Molitor-Braun, C.: Weighted group algebras on groups of polynomial growth. Math. Z. 245(4), 791-821 (2003)

21. Gabor, D.: Theory of communication. J. Inst. Electr. Eng. pp. 429-457 (1946)

22. Gelfand, I.: To the theory of normed rings. II. On absolutely convergent trigonometrical series and integrals. C. R. (Doklady) Acad. Sci. URSS (N.S.) 25, 570-572 (1939)

23. Gohberg, I., Kaashoek, M.A., Woerdeman, H.J.: The band method for positive and strictly contractive extension problems: an alternative version and new applications. Integral Equ. Oper. Theory 12(3), 343-382 (1989)

24. Gröchenig, K.: Aspects of Gabor analysis on locally compact abelian groups. In: Gabor Analysis and Algorithms, Appl. Numer. Harmon. Anal., pp. 211-231. Birkhäuser Boston, Boston, MA (1998)

25. Gröchenig, K.: Foundations of Time-Frequency Analysis. Appl. Numer. Harmon. Anal, Birkhäuser (2001)

26. Gröchenig, K.: A pedestrian's approach to pseudodifferential operators. In: Harmonic Analysis and Applications, Appl. Numer. Harmon. Anal., pp. 139-169. Birkhäuser Boston, Boston, MA (2006)

27. Gröchenig, K.: Time-frequency analysis of Sjöstrand's class. Rev. Math. Iberoam. 22(2), 703-724 (2006)

28. Gröchenig, K.: Wiener's Lemma: Theme and Variations. An Introduction to Spectral Invariance and Its Applications, pp. 175-234. Birkhäuser Boston, Boston, MA (2010)

29. Gröchenig, K., Klotz, A.: Noncommutative approximation: inverse-closed subalgebras and offdiagonal decay of matrices. Constr. Approx. 32(3), 429-466 (2010)

30. Gröchenig, K., Leinert, M.: Wiener's lemma for twisted convolution and Gabor frames. J. Am. Math. Soc. 17, 1-18 (2004)

31. Gröchenig, K., Strohmer, T.: Pseudodifferential operators on locally compact abelian groups and Sjöstrand's symbol class. J. Reine Angew. Math. 613, 121-146 (2007)

32. Hulanicki, A.: On the spectrum of convolution operators on groups with polynomial growth. Invent. Math. 17, 135-142 (1972)

33. Jakobsen, M.S.: On a (no longer) New Segal Algebra: A Review of the Feichtinger Algebra. J. Fourier Anal. Appl. 24(6), 1579-1660 (2018)

34. Jakobsen, M.S., Lemvig, J.: Density and duality theorems for regular Gabor frames. J. Funct. Anal. 270(1), 229-263 (2016)

35. Jakobsen, M.S., Luef, F.: Duality of Gabor frames and Heisenberg modules. J. Noncommut. Geom. 14(4), 1445-1500 (2020)

36. Kaiser, G.: A Friendly Guide to Wavelets. Birkhäuser Boston Inc, Boston, MA (1994)

37. Kreisel, M.: Gabor frames for quasicrystals, $K$-theory, and twisted gap labeling. J. Funct. Anal. 270(3), 1001-1030 (2016)

38. Kurbatov, V.G.: Algebras of difference and integral operators. Funktsional. Anal. i Prilozhen. 24(2), 87-88 (1990)

39. Leptin, H.: Darstellungen verallgemeinerter $L^{1}$-Algebren. Invent. Math. 5, 192-215 (1968)

40. Li, H.: Compact group automorphisms, addition formulas and Fuglede-Kadison determinants. Ann. Math. (2) 176(1), 303-347 (2012)

41. Losert, V.: On the structure of groups with polynomial growth. II. J. London Math. Soc. (2) 63(3), 640-654 (2001)

42. Ludwig, J.: A class of symmetric and a class of Wiener group algebras. J. Funct. Anal. 31(2), 187-194 (1979)

43. Luef, F.: Projective modules over non-commutative tori are multi-window Gabor frames for modulation spaces. J. Funct. Anal. 257(6), 1921-1946 (2009)

44. Luef, F.: Projections in noncommutative tori and Gabor frames. Proc. Am. Math. Soc. 139(2), 571-582 (2011)

45. Măntoiu, M.: Symmetry and inverse closedness for Banach *-algebras associated to discrete groups. Banach J. Math. Anal. 9(2), 289-310 (2015)

46. Măntoiu, M., Nistor, V.: Spectral theory in a twisted groupoid setting: spectral decompositions, localization and Fredholmness. Münster J. Math. 13(1), 145-196 (2020)

47. Murphy, G.J.: $C^{*}$-algebras and Operator Theory. Academic Press Inc, Boston, MA (1990)

48. Paterson, A.L.T.: Amenability. Mathematical Surveys and Monographs, vol. 29. American Mathematical Society, Providence, RI (1988)

49. Poguntke, D.: An example of a generalized completely continuous representation of a locally compact group. Studia Math. 105(2), 189-205 (1993) 
50. Rickart, C.E.: General Theory of Banach Algebras. The University Series in Higher Mathematics. D. van Nostrand Co., Inc., Princeton, N.J.-Toronto-London-New York, (1960)

51. Rieffel, M.A.: Projective modules over higher-dimensional noncommutative tori. Can. J. Math. 40(2), 257-338 (1988)

52. Samei, E., Wiersma, M.: Quasi-Hermitian locally compact groups are amenable. Adv. Math., 359, 106897, 25 (2020)

53. Schweitzer, L.B.: A short proof that $M_{n}(A)$ is local if $A$ is local and Fréchet. Int. J. Math. 3, 581-589 (1992)

54. Sjöstrand, J.: Wiener type algebras of pseudodifferential operators. In: Séminaire Sur Les Équations Aux Dérivées Partielles, 1994-1995, pages Exp. No. IV, 21. École Polytech., Palaiseau, (1995)

Publisher's Note Springer Nature remains neutral with regard to jurisdictional claims in published maps and institutional affiliations. 